\title{
Blanket dynamics in upflow suspended bed
}

\author{
S.T. Su, R.M. Wu, D.J. Lee* \\ Chemical Engineering Department, National Taiwan University, Taipei 10617, Taiwan
}

Received 9 April 2003; received in revised form 4 September 2003; accepted 9 September 2003

\begin{abstract}
A one-dimensional model for the spatio-temporal variation of solids fraction in the blanket considering both the hydrodynamic dispersion and the convection effects was proposed in this study. The model equation was a wave equation whose solution presented shock-wave like characteristic. The model output predicted that the distribution of the solids fraction in the blanket would, firstly, evolve to a uniform distribution at a lower solids fraction. Afterward, the blanket would compact itself to another uniform distribution at a higher solids fraction. Experimental works with synthetic raw water coagulated with $\mathrm{PACl}$ suspended in a lab-scale suspended bed confirmed the theoretical predictions. The solids flux plot was constructed with underdosed, optimal and overdosed suspensions. Change in coagulant dose would affect the blanket height and the response time. The solids flux curve was shifted accordingly. The "optimal' operational condition for the upflow suspended bed that produced the lowest effluent turbidity was identified and discussed.
\end{abstract}

(C) 2003 Elsevier Ltd. All rights reserved.

Keywords: Blanket; Flocculation clarifier; Model; Experiment; Solids flux

\section{Introduction}

Flocculation clarifier has been widely used in clean water production since being introduced in the 1930s. Owing to their more efficient flocculation and better chance of making solid contacts, flocculation clarifiers can deal with a surface loading two to three times higher than conventional coagulation-sedimentation basins [1-4]. The Taiwan Water Supply Corporation (TWSC) installed flocculation clarifiers for drinking water production in the early 1990s, and flocculation clarifiers now supply over $70 \%$ of Taiwan's drinking water. The floc blanket acts as both a particle coagulator and a filter, and thus is essential for clarifiers to produce quality drinking water.

The settling velocity of the coagulated flocs and the assigned upflow velocity control the blanket stability $[5,6]$. Stringent operational control is required to prevent

\footnotetext{
*Corresponding author. Tel.: + 886-2-2362-5632; fax:+8862-2362-3040.

E-mail address: djlee@ccms.ntu.edu.tw (D.J. Lee).
}

sludge washout from the clarifiers [7]. However, the clarifiers used in Taiwan experienced frequent blanket carryover, indicating an unstable operation subjected to fluctuations in raw water quality. For instance, as Chen et al. [8] reported, the Ping-Tsan Water Works of TWSC located at northern Taiwan, which treated water using eight flat-bottom blanket clarifiers at a rate of $300,000-400,000 \mathrm{~m}^{3} \mathrm{~d}^{-1}$ and used polyaluminum chloride $(\mathrm{PACl})$ as the coagulant, completely lost its blanket during low-turbidity period. Chen et al. [9] hypothesized that the observed unstable blanket might be attributable to part of the organic matters existing in raw water that deteriorated the blanket. Lin et al. [10] indicated that the interactions between particles in blanket could be weakened by the presence of the humic acid in the raw water.

Ives [11] proposed a model for particle removal in the clarifier. This model nonetheless overestimated the particle removal efficiency of the clarifier and predicted an unrealistic solids concentration distribution in the blanket. Could [12] modeled the blanket by assuming that the change in blanket height $H(\mathrm{~m})$ is controlled by 
the upflow velocity $U\left(\mathrm{~m} \mathrm{~h}^{-1}\right)$ and the settling velocity $V_{\text {sett }}\left(\mathrm{m} \mathrm{h}^{-1}\right)$

$\frac{\mathrm{d} H}{\mathrm{~d} t}=U-V_{\text {sett }}$

where $t$ represents the process time (h). Apparently, if the blanket concentration were spatially uniformly distributed, Eq. (1) simply represents the mass balance of the particles in the blanket if the floc concentration does not change with the upflow velocity. Gregory et al. [5] commented that the removal rate of particles entering the clarifier depends upon the solids concentrations in the blanket. Hence, based on Could's model, Head et al. [6] modeled the removal of particle entering the blanket by applying the solids flux theory as follows:

$L \frac{\mathrm{d} X}{\mathrm{~d} t}=U\left(X_{\mathrm{i}}-X\right)-\left(k_{\mathrm{fl}}-c H X+v_{\mathrm{s}} X\right)$

where $L(\mathrm{~m})$ is the tank height, $X_{\mathrm{i}}\left(\mathrm{mgl}^{-1}\right)$ the inlet particle concentration, $c\left(\mathrm{mg} \mathrm{l}^{-1}\right)$ the floc concentration in blanket, $v_{\mathrm{s}}\left(\mathrm{m} \mathrm{h}^{-1}\right)$ the settling velocity of the primary particles, $k_{\mathrm{fl}}\left(\mathrm{h}^{-1}\right)$ the flocculation factor, and $z(\mathrm{~m})$ the upward coordinate. Notably, the concentration $c$ is not the concentration of dried solids, while the settling velocity $v_{\mathrm{s}}$ is not $V_{\text {sett }}$ in Eq. (1). This model assumed that the blanket is a perfectly mixed tank reactor. By assuming certain correlations between the floc concentration and the settling velocity $V_{\text {sett }}$, the time variation of particle concentration $X$ could be simulated numerically by solving Eqs. (1) and (2).

Head et al.'s model assumed a completely mixed blanket. As Chen et al. [13] reported, when blanket carryover occurred at the reduction in coagulant dose, a concentration "wave" was emerged from the tank bottom and moved upward. This occurrence yielded complete loss of the existing blanket, and suggested that the blanket model must account for the spatial distribution of solids fraction. Such an analysis does not exist in pertinent literature to the authors' knowledge.

In-depth understanding to the blanket dynamics in the flocculation clarifier is essential to better control of a stable blanket with satisfactory particle removal efficiency. We herein analyzed a batchwise, upflow suspended bed with a blanket suspended in an upflow stream. Firstly, a one-dimensional model describing the spatio-temporal distribution of solids fraction in the blanket was proposed. Then experiments were conducted to verify the model prediction. According to the theoretical findings and the experimental data, the solids flux plot was constructed with underdosed, optimal and overdosed suspensions. The "optimal' operational condition for upflow suspended bed was proposed in the text.

\section{The blanket model}

Fig. 1 schematically presents the system under investigation. The counterbalance between the buoyant weight of individual flocs (acting downward) and the hydrodynamic drag force induced by the upflow stream (acting upward) controls the blanket's stability. A simplified, one-dimensional model for the spatio-temporal variation of solids fraction in the blanket considering both the hydrodynamic dispersion and the convection effects could be stated as follows [13]:

$\frac{\partial C}{\partial t}=D \frac{\partial^{2} C}{\partial z^{2}}-\frac{\partial C\left\{U-V_{\text {sett }}\left(C, A V I, d_{f}, \cdots\right)\right\}}{\partial z}$,

where $C$ (dimensionless) is the solids fraction; $D$ $\left(\mathrm{m}^{2} \mathrm{~h}^{-1}\right)$ is the effective diffusivity of flocs in the blanket; and $t(h)$ is the process time. By doing so, the interactions between individual particles and the flocs were lumped into the settling velocity $V_{\text {sett }}$, which depends upon the solids fraction, the floc volume (AVI), the floc size, and many other process parameters. The term "solids fraction" $(C)$ adopted here is determined using weighing and drying, which differs from the "floc concentration" used by Gregory et al. [5], with the latter indicating the volume fraction of sediment after 30 -min setting. Field observation normally revealed a

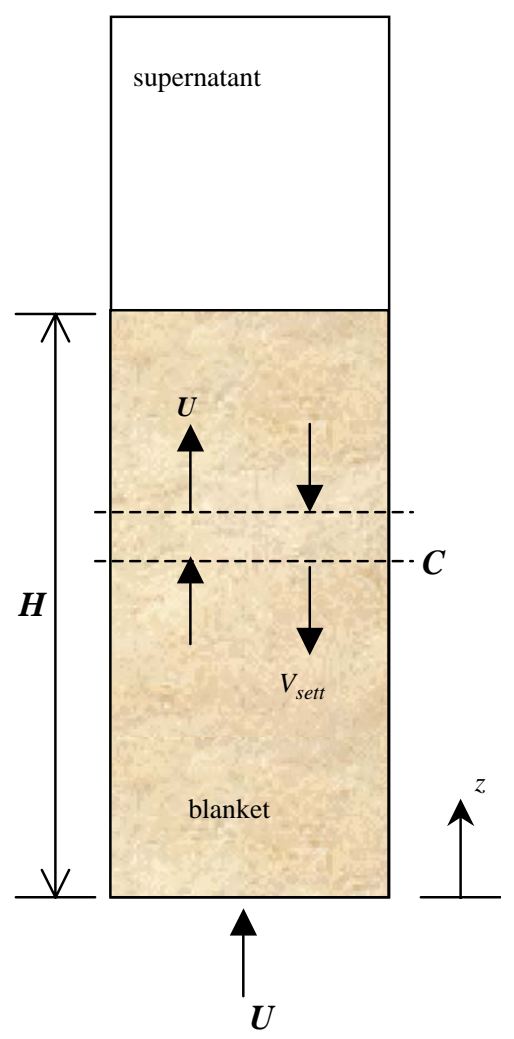

Fig. 1. The blanket model under investigation. 
rather uniform distribution of solids fraction within the floc blanket [6]. In addition, at steady-state operation, the blanket surface presented as a distinct surface from the clear supernatant. These observations suggested a negligible dispersion effect in the normal operation of clarification blanket process. At $D=0$ limit, the nodispersion approximation, Eq. (3) becomes a waveequation as follows:

$\frac{\partial C}{\partial t}+\left\{\frac{\partial\left(C\left(U-V_{\text {sett }}(C)\right)\right)}{\partial C}\right\} \frac{\partial C}{\partial z}=\frac{\partial C}{\partial t}+v(C) \frac{\partial C}{\partial z}$,

Eq. (4) has a shock-wave solution. $v(C)\left(\mathrm{m} \mathrm{h}^{-1}\right)$ in Eq. (4) is the characteristic velocity determined by the partial differentiation of the difference in solids fluxes $\left(C U-C V_{\text {sett }}\right)$. Therefore, the flocs existing at $z=z_{\mathrm{i}}$ and

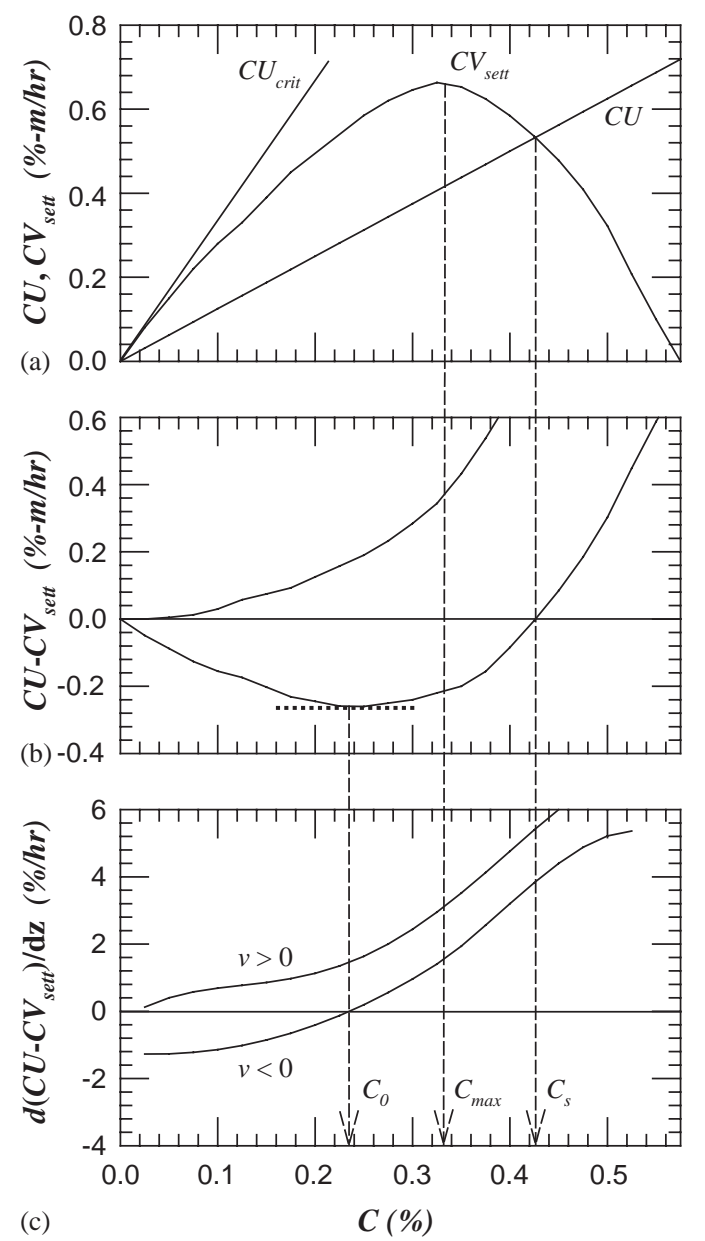

Fig. 2. Solids fluxes versus solids fraction plot. (a) The settling flux and the upflow flux curves. (b) The difference between settling and upflow solids fluxes. (c) The derivative of part (b), equivalent to the characteristic velocity of the investigated system. $V_{\text {sett }}=-9.04 C^{2}+0.08 C+2.88$, $U_{\text {crit }}=3.1 \mathrm{~m} / \mathrm{h}, \quad U=1.25 \mathrm{~m} / \mathrm{h}$. The concentration distribution for part (c) is $C=0.163 z^{-1}$. $t=t_{\mathrm{i}}$ would move upward or downward with a velocity of $v(C)$, following the trajectory stated as follows:

$z=z_{\mathrm{i}}+v\left(C\left(z_{\mathrm{i}}, t_{\mathrm{i}}\right)\right) t$,

where $C\left(z_{\mathrm{i}}, t_{\mathrm{i}}\right)$ is the floc concentration at $z=z_{\mathrm{i}}$ and $t=t_{\mathrm{i}}$. The arbitrarily distributed flocs at $t=t_{\mathrm{i}}$ would evolve according to the travel velocity of $v$.

Fig. 2a schematically presents the typical solids flux curves in the suspended bed with $V_{\text {sett }}$ as an empirical correlation of solids fraction, $V_{\text {sett }}=-9.04 C^{2}+0.08 C+2.88$, as an illustrative example. The settling flux $\left(C V_{\text {sett }}\right)$ would be zero at small or large $C$ limit, with a local maximum occurred at $C=C_{\max }\left(\mathrm{mgl}^{-1}\right)$. The upflow flux is a straight line in Fig. 2a of slope $U$. Then the difference in solids fluxes and its slope could be demonstrated in Figs. 2b and c, respectively. (In Fig. 2c, the spatial distribution of solids fraction is taken as $C=0.163 z^{-1}$ for illustration purpose.) Notably, the characteristic velocity would be negative when $C$ is small, and would approach a positive constant at large $C$ limit. At $C=C_{0}$, which is the local maximum on curve in Fig. 2b, $v\left(C_{0}\right)=0$. Apparently, $C_{0}<C_{\max }$, and $C_{0} \rightarrow C_{\max }$ when $U \rightarrow 0$.

According to Eq. (5), $v(C)$ is positive when $C>C_{0}$. The vertical position with $C=C\left(z_{\mathrm{i}}, t_{\mathrm{i}}\right)$ would move upward according to Eq. (5). When $C<C_{0}$, the vertical position with $C=C\left(z_{\mathrm{i}}, t_{\mathrm{i}}\right)$ would move downward since $v(C)<0$. The local solids fraction distribution with $v\left(C_{0}\right)=0$ would not change with time. This evolution process is schematically demonstrated in Fig. 3. The solids fraction distribution in the blanket would hence converge to a discontinuous solution, or a shock wave, at $C=C_{0}$. Above the interface a supernatant existed with $C \approx 0$.

Apparently $C U>C V_{\text {sett }}$ at $C=C_{0}$ (Fig. 2b). After the solids fraction had reached the shock-wave solution at $C=C_{0}$, subsequently, the blanket would evolve to

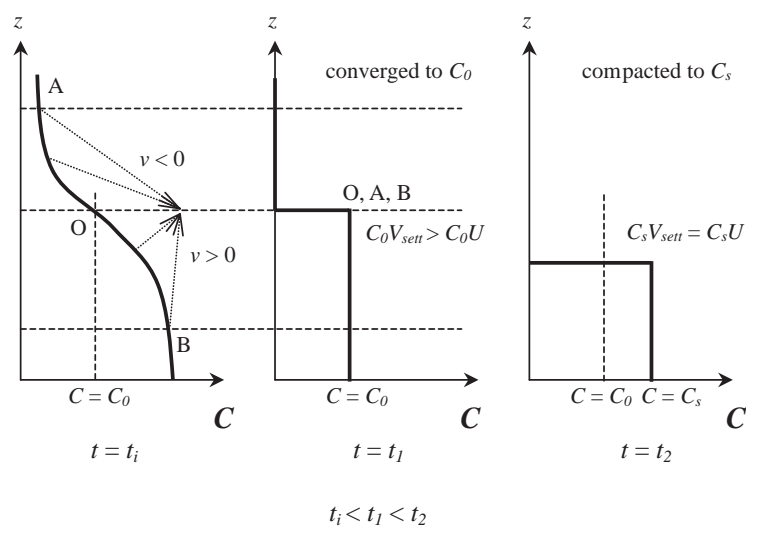

Fig. 3. The schematics of the evolution process of the blanket. $\left(t=t_{i}\right)$ Blanket in an initial distribution; $\left(t=t_{1}\right)$ blanket converges to a uniform distribution at $C_{0} ;\left(t=t_{2}\right)$ blanket compacts itself to the final, uniform distribution at $C_{\mathrm{S}}$. 
another shock-wave solution at $C=C_{\mathrm{S}}$ (Fig. 2a), determined by the intersection of $C U$ and $C V_{\text {Sett }}$ curves. Restated, since $C_{0}<C_{\mathrm{S}}$, the blanket initially formed at $C_{0}$ would compact to a shock-wave-like distribution at $C=C_{\mathrm{S}}$. The time required for the blanket to reach this steady-state distribution is not available from Eq. (4) since it is no longer valid once the shock-wave distribution has formed at $C=C_{0}$.

A critical velocity could be defined as $U_{\text {crit }}=$ $\lim _{C \rightarrow 0}\left(\partial\left(C V_{\text {sett }}\right) / \partial C\right)\left(\mathrm{m} \mathrm{h}^{-1}\right)$. The straight line of $C U_{\text {crit }}$ was also indicated in Fig. 2. At $U>U_{\text {crit }}$ there would be no steady-state solution of $C$, indicating the complete loss of blanket.

\section{Experimental}

The synthetic raw water was prepared by mixing prescribed amount of UK ball clay powders and $10^{-2} \mathrm{~N}$ $\mathrm{NaClO}_{4}$ solution to a solid weight fraction of $0.16 \%$, giving a turbidity of $200 \mathrm{NTU}$. The alkalinity was adjusted by adding $\mathrm{NaHCO}_{3}$ salt to $100 \mathrm{mgl}^{-1}$ equivalent. The $\mathrm{pH}$ of suspension was adjusted using $\mathrm{HClO}_{4}$ and $\mathrm{NaOH}$ to 7 . The suspension was coagulated in a jar tester. The 1000-ppm PACl solution, with $11 \%$ available $\mathrm{Al}_{2} \mathrm{O}_{3}$, was slowly injected into the stirred suspension at $90 \mathrm{rpm}$ for $1.5 \mathrm{~min}$, and then at $50 \mathrm{rpm}$ for $8.5 \mathrm{~min}$. The residual turbidities of supernatant of 2-h settled coagulated samples were measured, from which the optimal $\mathrm{PACl}$ dose was determined for the present synthetic raw water as $16 \mathrm{ppm}$ as $\mathrm{Al}$.

The sediment of the coagulated raw water was carefully collected, and was gradually poured into a cylindrical column of diameter $5.7 \mathrm{~cm}$ and height of $87 \mathrm{~cm}$ with care. Fig. 4 schematically depicts the fluidized bed used in all tests. A low upflow velocity was applied continuously from the column bottom during the sediment pouring. Preliminary test revealed

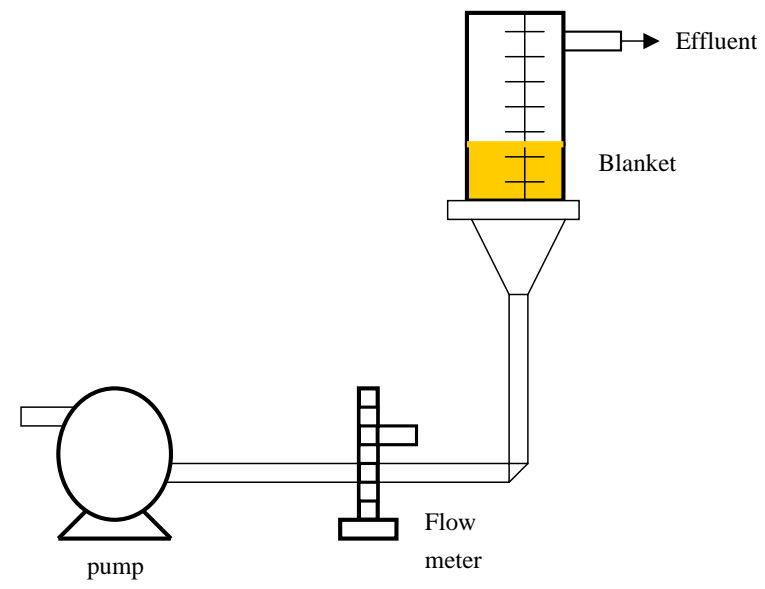

Fig. 4. The schematics of the fluidized bed in the test. that this low upflow velocity was essential to keep the sediment in a suspended state for getting reproducible data. The compaction of sediment at column bottom would yield irreversible change in its structure [14-16]. This occurrence would hinder sediment resuspension with the upflow stream applied afterward.

In the dynamic tests, a digital camera recorded the position of the interface height, with particular attention to the full suspension of the entire blanket without compacted sediment layer to form at the column bottom. It took several minutes to four hours for the blanket to be equilibrated with the water flow, depending on the PACl dose and the upflow velocity. Afterwards the upflow velocity was turned off and the blanket was settled freely.

In the steady-state tests, only the equilibrium heights of blanket interface were recorded at various upflow velocities. A turbidimeter (HACH Model 2100 AN) measured the turbidities of the effluent from the suspended bed.

\section{Results and discussion}

\subsection{Dynamic test}

A typical test for a slurry coagulated with $\mathrm{PACl}=16 \mathrm{ppm}$ as $\mathrm{Al}$ and upflow velocity of $2.49 \mathrm{~m} \mathrm{~h}^{-1}$ was demonstrated in Fig. 5. After the slurry was poured into the column (with $U=2.49 \mathrm{~m} \mathrm{~h}^{-1}$ ), it took about $20 \mathrm{~min}$ for a distinct supernatant-blanket interface to appear at $H=63 \mathrm{~cm}$. Then the blanket gradually settled, and the height reached a plateau value at $H=55 \mathrm{~cm}$ during $t=60-100 \mathrm{~min}$ (region $\mathrm{AB}$ ). Afterwards this blanket started to settle again, and finally equilibrated with the upflow velocity at $H=42 \mathrm{~cm}$ (point $D$,

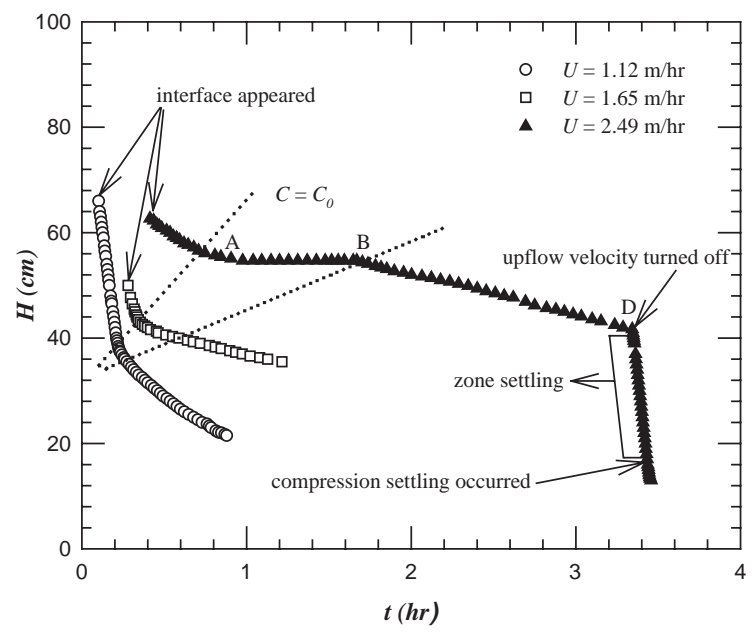

Fig. 5. Dynamic test with suspension coagulated at $\mathrm{PACl}=16 \mathrm{ppm}$ as $\mathrm{Al}$. 
coincided with the equilibrium blanket height from independent test). Visual observation indicated that the solids phase in the blanket was fully suspended and the solids fraction could be taken as uniformly distributed in the column. Head et al. [6] and Chen et al. [8,9,13] also demonstrated that the solids fraction distribution is rather uniform in the full-scale blanket clarifier. In this regard, the average solids fraction in the blanket could be estimated $0.22 \%$ (namely $C_{0}$ ) at region $\mathrm{AB}$, and as $0.33 \%$ (namely $C_{\mathrm{S}}$ ) at point $D$. This observation corresponds to the model prediction: the randomly distributed flocs would first converge to a uniform distribution at lower solids fraction $(0.22 \%$ in this case), then the blanket would compact itself to another uniform distribution at higher solids fraction $(0.33 \%$ in this case).

When the upflow velocity was turned off, the blanket settled to a height of $13 \mathrm{~cm}$ in $5 \mathrm{~min}$. Notably, the $H-t$ curve reveals a linear character over $H=39-18 \mathrm{~cm}$, presenting a zone-settling characteristic. The flocs started to physically contact each other only at $H<18 \mathrm{~cm}$, corresponding to $C=0.77 \%\left(C_{t}\right)$. When flocs physically contacted with each other, a yield stress could be built up in the "network" among the flocs, which could produce a plateau region similar to those noted for regime $\mathrm{AB}$. Since both $C_{0}$ and $C_{\mathrm{S}}$ were much smaller than $C_{t}$, therefore, the region $\mathrm{AB}$ and point $D$ in Fig. 5 were in a suspended state. Restated, the emergence of the plateau region $\mathrm{AB}$ was attributable to the shockwave solution of Eq. (4) but to the possible network structure made by floc contacts.

Fig. 5 also shows the suspended tests at $U=1.12$ or $1.65 \mathrm{~m} \mathrm{~h}^{-1}$, respectively. As expected, a lower upflow velocity yielded earlier appearance of the blanket interface, and subsequently, the blanket settled faster than those at higher upflow velocity. Moreover, the plateau region $\mathrm{AB}$ was not as obvious for $U=1.12$ or $1.65 \mathrm{~m} \mathrm{~h}^{-1}$ as that noted at $2.49 \mathrm{~m} \mathrm{~h}^{-1}$ test. Only a dramatic change in slope appeared on $H-t$ curve at $H=37$ or $42 \mathrm{~cm}$, as indicated by arrows in Fig. 5. The corresponding solids fractions $\left(C_{0}\right)$ were $0.40 \%$ or $0.35 \%$, respectively. Since these heights were higher than $18 \mathrm{~cm}\left(C_{t}\right)$, as mentioned above, the slow-down phenomenon was not attributable to the compaction of particles in the sediment. Finally, the interface approached $H=22$ or $30 \mathrm{~cm}$ at $U=1.12$ or $1.65 \mathrm{~m} \mathrm{~h}^{-1}$, corresponding to $C_{\mathrm{S}}=0.63 \%$ or $0.46 \%$, respectively.

Similar blanket dynamics were also noted for tests with $\mathrm{PACl}=12 \mathrm{ppm}$ (underdosed) or $20 \mathrm{ppm}$ (overdosed) as $\mathrm{Al}$ (data not shown). Restated, after a period of settling a blanket would form (and may stay stationary for a period of time) in the column, whose solids fraction revealed a shock-wave like distribution $\left(C=C_{0}\right)$. Afterwards the blanket settled again to the equilibrium height at $C=C_{\mathrm{S}}$. Experimental findings thus verified the model output developed in the previous section. The plateau regime $(\mathrm{AB})$ existed commonly in all tests, whose blanket height increased with upflow velocity and/or $\mathrm{PACl}$ dose.

\subsection{Steady-state test}

The steady-state tests measured the equilibrium positions of blanket interface and the turbidities of effluent at various upflow velocities (Fig. 6). The interface height increased with increasing upflow

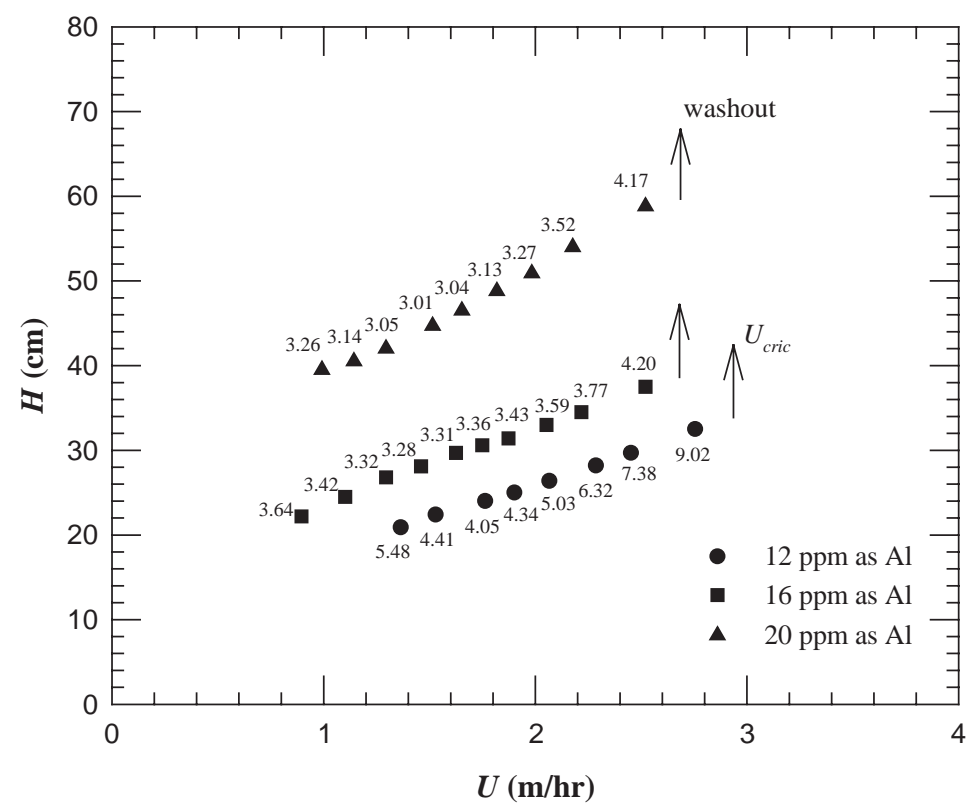

Fig. 6. Equilibrium heights and effluent turbidities of coagulated suspensions at various upflow velocities. Steady-state tests. 
velocity and/or $\mathrm{PACl}$ dose. At $\mathrm{PACl}=12 \mathrm{ppm}$ as $\mathrm{Al}$ which could not sufficiently coagulate the suspension, the resulting blanket was compact. As shown in Fig. 6 the blanket heights were low. But the corresponding effluent turbidities ranged 4.03-9.02 NTU. Fine particles and large fragments flew out with the effluent. The interface became blurred at $U=2.75 \mathrm{~m} \mathrm{~h}^{-1}$. At $U=2.85 \mathrm{~m} \mathrm{~h}^{-1}$, the blanket was largely carried out, and finally completely lost. This velocity was a critical velocity $U_{\text {crit }}$ mentioned earlier.

When the particles were sufficiently coagulated at $16 \mathrm{ppm}$ as $\mathrm{Al}$, the blanket had a loose structure and was easily expanded with the upflow stream. The equilibrium heights of the blanket were much larger than the 12-ppm tests. The corresponding effluent turbidities were also low (3.31-4.20 NTU). At an upflow velocity similar to $U_{\text {crit }}$ of 12-ppm test, the carryover of blanket occurred. Restated, to coagulate suspension at optimal dose (determined by gravity settling) would produce quality effluent, but could not effectively increase the operational range of the blanket.

Adding $\mathrm{PACl}$ at $20 \mathrm{ppm}$ as $\mathrm{Al}$ into suspension would further increase the equilibrium heights of blanket. The corresponding effluent turbidity (3.01-4.17) was slightly lower than the 16-ppm test. Visual observation noted that in effluent many large fragments containing many coagulated flocs had flowed out with the effluent. The corresponding critical velocity $U_{\text {crit }}$ for 20 -ppm test was close to those for 12 or 16-ppm tests.

\subsection{Solids flux plot}

According to the obtained $C_{0}$ and $C_{\mathrm{S}}$ data at various $U$ 's, and the $U_{\text {crit }}$ data noted in the steady-state tests, the corresponding solids flux curves at coagulant dose of $16 \mathrm{ppm}$ as $\mathrm{Al}$ could be constructed. Since the blanket interface would be blurred at high-upflow velocities, the solids fluxes at low solids fraction region could not be accurately estimated. Meanwhile, the straight line of $C U_{\text {crit }}\left(U_{\text {crit }}=2.75 \mathrm{~m} \mathrm{~h}^{-1}\right.$ in this case $)$ represents the asymptotic behavior of the solids flux at $C \rightarrow 0$ limit, which was used to bound the left margin of the solids flux curve (line OL). Hence, the complete solids flux curve could be approximated by curve $O C_{\max } \mathrm{M}$ in Fig. 7. As Fig. 2a depicts, the solids flux curve exhibited an increasing-decreasing characteristic, with its maximum at $C=C_{\max }$ and $U=U_{\max }$. Theoretically, one could operate his blanket up to $U_{\text {crit }}$, which is the tangent line for the solids flux curve at $C \rightarrow 0$ limit. However, as experiments revealed, the blanket became blurred at an upflow velocity lower than $U_{\text {crit }}$. Without theoretical justification, Gregory et al. [5] recommended to operate the blanket at $C=C_{\max }$ based on field observation. Since $U_{\max }<U_{\text {crit }}$, the Gregory's proposal represented a convenient index to field operation. However, it is not

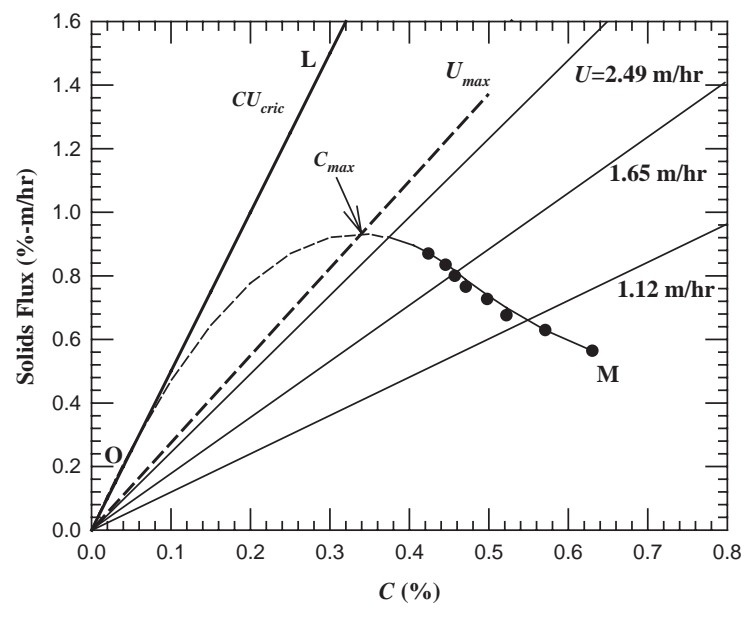

Fig. 7. The solids flux curves of suspension coagulated at $16 \mathrm{ppm}$ as Al. Solid circles: data from steady-state test; open triangles and open squares: $C_{\mathrm{S}}$ and $C_{0}$ data from dynamic tests.

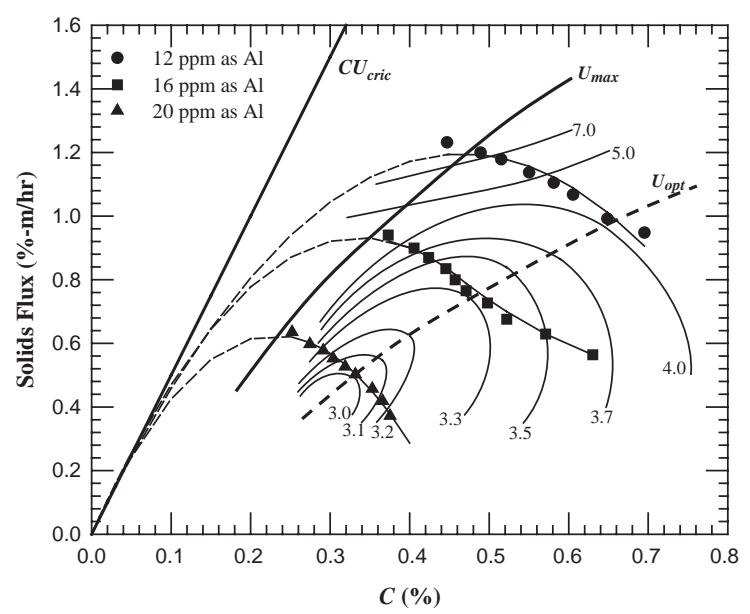

Fig. 8. The solids flux curves and the effluent turbidities of coagulated suspensions at various $\mathrm{PACl}$ doses.

necessary the "optimal" operational condition since no theoretical justification was made to support it.

Fig. 8 depicts the solids flux curves for tests with $\mathrm{PACl}=12,16$, and $20 \mathrm{ppm}$ as $\mathrm{Al}$, where the dose of $16 \mathrm{ppm}$ was the "optimal dose" determined by the supernatant turbidity after gravity settling (Fig. 1). The solids flux curve shifts downwards and leftwards at higher $\mathrm{PACl}$ dose, and vice versa. This occurrence corresponds to the loose blanket structure noted for high PACl tests (Fig. 6). Also, since the $U_{\text {crit }}$ 's for various $\mathrm{PACl}$ doses are close, all curves exhibited a similar asymptote at the $C \rightarrow 0$ limit. The contours of the turbidities in effluent were also demonstrated in the figure. Notably, for a given PACl dose the effluent turbidity reach a local minimum at a specific upflow 
velocity lower than $U_{\max }$, indicating as $U_{\text {opt }}$ in Fig. 8 . Restated, if the minimum effluent turbidity is considered as "optimal", then the operation should be set at $U_{\text {opt }}$ rather than at $U_{\max }$. Also interestedly, the turbidity of effluent at $U_{\text {opt }}$ decreases with increasing $\mathrm{PACl}$ dose, although the magnitude of decline decreases at dose larger than $16 \mathrm{ppm}$ as $\mathrm{Al}$. Hence, the optimal dose determined by conventional jar tests corresponds to the "optimal dose" for the current suspended bed test. This observation, however, should be considered preliminary at this stage. Further experimental verification was required.

\subsection{Effects of sediment compaction}

In Section 3 we emphasized the significant role of the applied upflow velocity for the data reproducibility. Fig. 9 depicts an example with $\mathrm{PACl}=16 \mathrm{ppm}$ as $\mathrm{Al}$. Before testing the blanket was suspended at $U=1.0 \mathrm{~m} \mathrm{~h}^{-1}$, with its equilibrium height being $28.2 \mathrm{~cm}$. Then at point $O$ the upflow velocity was increased to $2.0 \mathrm{~m} \mathrm{~h}^{-1}$, which reached a new equilibrium height of $39.6 \mathrm{~cm}$. At point $A$ the upflow velocity was reduced to $1.0 \mathrm{~m} \mathrm{~h}^{-1}$, while the blanket resumed its original height of $28.2 \mathrm{~cm}$. At point $B$ the upflow velocity was turned off. The blanket settled freely and the compaction occurred at $H=15 \mathrm{~cm}$. Without sufficient sediment compaction, at point $C$ the upflow velocity of $1.0 \mathrm{~m} \mathrm{~h}^{-1}$ was provided to suspend the blanket. The equilibrium height became $23 \mathrm{~cm}, 4.6 \mathrm{~cm}$ lower than that at point $O$ or point $B$, showing an irreversible change in blanket structure. To re-suspend the blanket at $U=2.1 \mathrm{~m} \mathrm{~h}^{-1}$ yielded an equilibrium blanket height of $35 \mathrm{~cm}$, a $4.5 \mathrm{~cm}$ difference than its corresponding state at point $A$. Hence, as stated in previous sections, to keep the blanket in a suspended state is essential for reproducible data collection.

\section{Conclusions}

We proposed a simplified one-dimensional wave equation to model the dynamic characteristics of the floc blanket in an upflow suspended bed. Since the solids fraction would evolve along the characteristic velocity determined by the difference between upflow and downward solids fluxes, the blanket would first converge to a uniform distribution at a lower solids concentration, then it would compact itself to a new, uniform distribution at a higher solids fraction. Experiments with synthetic raw water using polyaluminum chloride (PACl) as coagulant confirmed the model prediction. Restated, it was noted that the blanket could stay stationary at a level higher than the final equilibrium height with or without an upflow. The solids fraction at equilibrium heights together with the critical upflow velocity that carried over the blanket constructed the solids flux curves with suspensions conditioned at underdosed, optimal (based on jar test results), and overdosed coagulant. The solids flux curve exhibited an increasing-decreasing characteristic, with its maximum at $C=C_{\max }$ and $U=U_{\max }$. We noted that to operate at $C_{\max }$ point, as Gregory et al. [5] proposed, is a workable, but not "optimal" criterion if the minimum effluent turbidity were required. Instead, an upflow velocity at $U_{\text {opt }}$, which is less than $U_{\max }$, was identified to be the optimal operative velocity for the suspended bed. The optimal dose of coagulant determined by conventional jar tests corresponds to the "optimal dose" for the

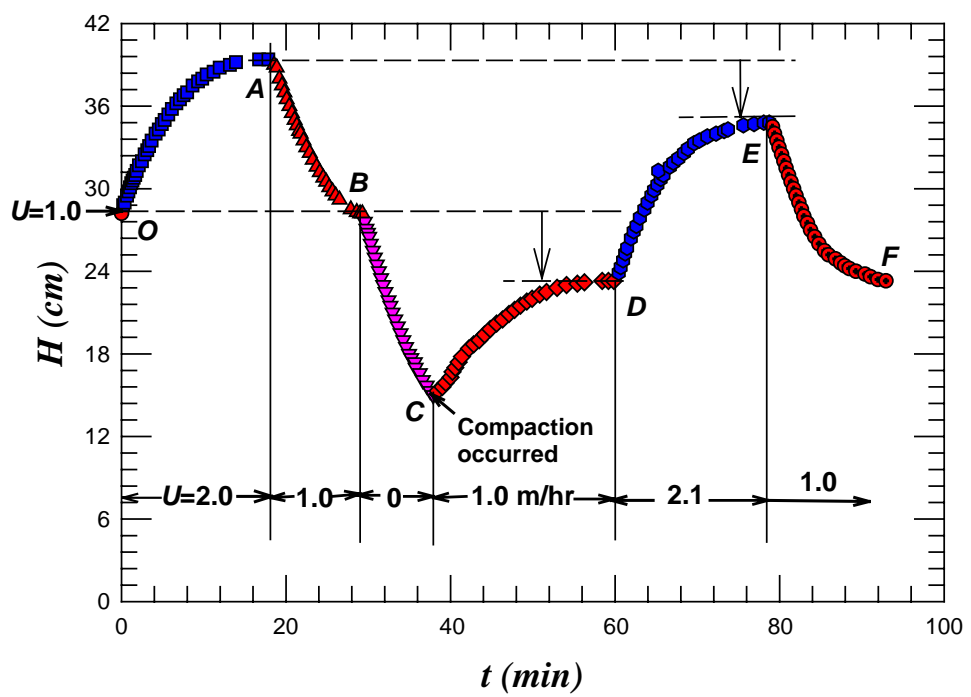

Fig. 9. Effects of sediment compaction on the observed dynamics of blanket height in suspended bed. $16 \mathrm{ppm}$ as Al. 
current suspended bed test. We also demonstrated that the compaction of collected blanket samples would yield experimental artifacts because of the build-up of certain networks among the particles.

\section{Acknowledgements}

The National Science Council, ROC, financially supported this work.

\section{References}

[1] Kawamura S. Integrated design of water treatment facilities. New York: Wiley; 1991.

[2] Masschelein WJ. Unit processes in drinking water treatment. New York: Marcel Dekker; 1992.

[3] Stevenson DG. Water treatment unit process. London: Imperial College Press; 1997.

[4] Edzwald JK, Ives KJ, Janssens JG, McEwen JB, Wiesner MR. Treatment process selection for particle removal. New York: AWWRF/IWSA; 1999 [chapter 5].

[5] Gregory R, Head R, Graham NJD. Blanket solids concentration in floc blanket clarifiers. Proceedings of the Gothenburg Symposium. Edinburgh, 1996.

[6] Head R, Hart J, Graham NJD. Simulating the effect of blanket characteristics on the floc blanket clarification process. Water Sci Technol 1997;36(4):77-82.
[7] AWWA/ASCE. Water treatment plant design. New York: McGraw-Hill; 1990 [chapter 7].

[8] Chen LC, Sung SS, Lin WW, Lee DJ, Huang C, Juang RS, Chang HL. Observations of blanket characteristics in fullscale floc blanket clarifiers. Water Sci Technol 2003;47(1): 197-204.

[9] Chen LC, Lin WW, Sung SS, Chung HY, Wang ZC, Wu RM, Lee DJ, Huang C, Juang RS, Peng XF, Chang HL. Treating high-turbidity water using full-scale floc blanket clarifiers. J Environ Eng ASCE 2003; in press.

[10] Lin WW, Sung SS, Lee DJ, Chen YP, Chen DS, Lee SF. Coagulation of humic-kaolin-PACl aggregates. Water Sci Technol 2003;47(1):145-52.

[11] Ives KJ. Theory of operation of sludge blanket clarifier. Proc Inst Civ Eng 1968;39:243-60.

[12] Could BW. Upflow clarifiers-flow flexibility related to concentrator size. Effluent Water Treat J 1974;14(11): 621-31.

[13] Chen LC, Sung SS, Lin WW, Lee DJ. Spatial instability of blanket in full-scale blanket clarifiers. J Chin Inst Chem Eng 2003;34(4):447-56.

[14] Chu CP, Lee DJ. Solids fluxes in consolidating sediments. J Chin Inst Chem Eng 2001;32:547-54.

[15] Chu CP, Ju SP, Lee DJ, Mohanty KK. Batch sedimentation of slurries: effects of initial concentrations. J Colloid Interface Sci 2002;245:178-86.

[16] Chu CP, Ju SP, Lee DJ, Tiller FM, Mohanty KK, Chang YC. Batch settling of flocculated clay slurry. Ind Eng Chem Res 2002;41:1227-33. 